\title{
Several Treatments on Nonconforming Element Failed in the Strict Patch Test
}

\author{
Xiao-Ming Chen, ${ }^{1}$ Song Cen, ${ }^{2,3,4}$ Yun-Gui Li, ${ }^{1}$ and Jian-Yun Sun ${ }^{1}$ \\ ${ }^{1}$ China State Construction Technical Center, Beijing 101300, China \\ ${ }^{2}$ Department of Engineering Mechanics, School of Aerospace, Tsinghua University, Beijing 100084, China \\ ${ }^{3}$ High Performance Computing Center, School of Aerospace, Tsinghua University, Beijing 100084, China \\ ${ }^{4}$ Key Laboratory of Applied Mechanics, School of Aerospace, Tsinghua University, Beijing 100084, China \\ Correspondence should be addressed to Song Cen; censong@mail.tsinghua.edu.cn
}

Received 19 June 2013; Accepted 23 July 2013

Academic Editor: Chenfeng Li

Copyright ( 2013 Xiao-Ming Chen et al. This is an open access article distributed under the Creative Commons Attribution License, which permits unrestricted use, distribution, and reproduction in any medium, provided the original work is properly cited.

For nonconforming finite elements, it has been proved that the models whose convergence is controlled only by the weak form of patch tests will exhibit much better performance in complicated stress states than those which can pass the strict patch tests. However, just because the former cannot provide the exact solutions for the patch tests of constant stress states with a very coarse mesh (strict patch test), their usability is doubted by many researchers. In this paper, the non-conforming plane 4-node membrane element AGQ6-I, which was formulated by the quadrilateral area coordinate method and cannot pass the strict patch tests, was modified by three different techniques, including the special numerical integration scheme, the constant stress multiplier method, and the orthogonal condition of energy. Three resulting new elements, denoted by AGQ6M-I, AGQ6M-II, and AGQ6M, can pass the strict patch test. And among them, element AGQ6M is the best one. The original model AGQ6-I and the new model AGQ6M can be treated as the replacements of the well-known models Q6 and QM6, respectively.

\section{Introduction}

In order to overcome the over-stiffness problem existing in conforming finite elements and improve their performances in regular and distorted meshes, the appearance of the nonconforming elements seems to be inevitable, such as the famous 4-node quadrilateral plane element Q6 proposed by Wilson et al. [1], the rectangular thin plate bending element ACM proposed by Melosh [2], and the triangular thin plate bending element BCIZ proposed by Bazeley et al. [3]. Since the conforming requirements along element edges are relaxed, the stiffness matrices of these elements become much softer. So, they can exhibit better performance than usual conforming element models in most cases. Furthermore, the construction procedures of these formulations are also easier. But, from the viewpoint of the variational principle, these elements violate the principle of minimum potential energy, which leads to that most of them cannot pass the strict patch tests, that is, their convergence cannot be guaranteed.
The patch test proposed by Irons et al. $[3,4]$ has been taken as a standard for examining the convergence of the nonconforming elements for a long time. In such test, the nonconforming elements are required to produce exact solutions for a constant strain/stress patch with a very coarse mesh. Although it has been adopted very broadly, there are still some further discussions [5-8] on it. The patch test usually includes two types: the "strict" form and the "weak" form [9]. The strict patch test requests that the element can yield exact solutions under fixed coarse meshes and has been accepted by most researchers, while the weak form only requires the element to provide convergent solution for constant stress/strain problem as the mesh is repeatedly subdivided. Actually, the thought of the weak form of the patch test is more consistent with the concept of convergence.

Many efforts have been made for developing 4-node nonconforming plane elements without any problem in convergence, such as the element QM6 proposed by Taylor et al. [10], QP6 by Wachspress [11], NQ6 by Pian and Wu [12], the 
generalized conforming element GC-Q6 by Long and Huang [13], the quasiconforming element QC6 by Chen and Tang [14], the hybrid-stress element P-S by Pian and Sumihara [15], and the Hamilton hybrid-stress element $\mathrm{HH} 4$ by Cen et al. [16]. All these elements can pass the strict form of patch test and possess much better performance than usual 4-node conforming isoparametric element Q4. In 2004, Chen et al. [17] formulated a 4-node nonconforming quadrilateral plane element AGQ6-I by using the quadrilateral area coordinates $[18,19]$. This model exhibits excellent performance and is quite insensitive to mesh distortions in higher order problems. For example, it can keep the same high precision for famous MacNeal's beam bending problem [20] using various distorted meshes, while other models cannot achieve this. This distinguished property can also be shown in nonlinear problems [21]. Furthermore, the explicit expressions of the element stiffness matrix can be obtained according to the integral formulae of the quadrilateral area coordinates [22], which can bring better computation efficiency. So, the element AGQ6-I attracts other researchers to make further developments and applications [23-25]. On the other hand, this element failed in strict patch test and can only pass the weak patch test. So, its convergence also arose some discussions [26-28].

In this paper, three treatments were tried to improve the convergence of the element AGQ6-I, including the special numerical integration scheme, the constant stress multiplier method, and the orthogonal condition of energy. Three resulting new elements, denoted by AGQ6M-I, AGQ6MII, and AGQ6M, respectively, can pass the strict patch test. Numerical examples show that, among the three new models, the element AGQ6M is the best one. The original model AGQ6-I and the new model AGQ6M can be treated as the replacements of the well-known models Q6 and QM6, respectively.

\section{Brief Reviews on Element AGQ6-I}

The 4-node quadrilateral plane membrane element AGQ6-I was formulated by the quadrilateral area coordinate methods [17]. Its displacement fields contain two parts: a generalized conforming low-order displacement part which is interpolated by the nodal displacements, and an additional secondorder nonconforming part.

2.1. Low-Order Displacement Interpolation Formulae. For a 4node quadrilateral plane element, the degrees of freedom can be expressed by the nodal displacement vector:

$$
\mathbf{q}=\left[\begin{array}{llllllll}
u_{1} & v_{1} & u_{2} & v_{2} & u_{3} & v_{3} & u_{4} & v_{4}
\end{array}\right]^{\mathrm{T}} .
$$

Then, the low-order displacement fields can be assumed as

$$
\begin{aligned}
u^{0}= & \alpha_{1}+\alpha_{2}\left(L_{3}-L_{1}\right)+\alpha_{3}\left(L_{4}-L_{2}\right) \\
& +\alpha_{4}\left(L_{3}-L_{1}\right)\left(L_{4}-L_{2}\right), \\
v^{0}= & \beta_{1}+\beta_{2}\left(L_{3}-L_{1}\right)+\beta_{3}\left(L_{4}-L_{2}\right) \\
& +\beta_{4}\left(L_{3}-L_{1}\right)\left(L_{4}-L_{2}\right)
\end{aligned}
$$

in which $L_{i}(i=1,2,3,4)$ are quadrilateral area coordinates and have been defined in [18]. In order to solve the undetermined parameters $\alpha_{i}$ and $\beta_{i}(i=1,2,3,4)$, two nodal conforming conditions and two generalized conforming conditions along the perimeter $\partial A^{e}$ of the element are introduced for both $u^{0}$ and $v^{0}$. Taking $u^{0}$ as the example, these conditions are as follows:

$$
\begin{gathered}
\sum_{i=1}^{4}\left(u^{0}-\tilde{u}\right)_{i}=0, \\
\sum_{i=1}^{4}\left(u^{0}-\tilde{u}\right)_{i} \xi_{i} \eta_{i}=0, \\
\oint_{\partial \mathrm{A}^{e}} l\left(u^{0}-\tilde{u}\right) \mathrm{d} s=0, \\
\oint_{\partial \mathrm{A}^{e}} m\left(u^{0}-\tilde{u}\right) \mathrm{d} s=0 .
\end{gathered}
$$

Thus, the shape functions of the low-order displacement fields can be obtained:

$$
N_{i}^{0}=-\frac{1-g_{i}}{2}+L_{i}+L_{i+1}+\xi_{i} \eta_{i}\left(1-g_{i}\right) \bar{P}, \quad(i=1,2,3,4)
$$

with

$$
\bar{P}=\frac{1}{g_{2} g_{4}+g_{1} g_{3}}\left[\left(L_{3}-L_{1}\right)\left(L_{4}-L_{2}\right)-\frac{1}{2}\left(g_{2} g_{4}-g_{1} g_{3}\right)\right]
$$

in which $g_{i}(i=1,2,3,4)$ are the shape parameters for a quadrangle and have been defined in [18]. by

Then, the corresponding strain matrix can be expressed

$$
\mathbf{B}_{q}=\left[\begin{array}{llll}
\mathbf{B}_{q 1} & \mathbf{B}_{q 2} & \mathbf{B}_{q 3} & \mathbf{B}_{q 4}
\end{array}\right]
$$

where

$$
\mathbf{B}_{q i}=\left[\begin{array}{cc}
\frac{\partial N_{i}}{\partial x} & 0 \\
0 & \frac{\partial N_{i}}{\partial y} \\
\frac{\partial N_{i}}{\partial y} & \frac{\partial N_{i}}{\partial x}
\end{array}\right], \quad(i=1,2,3,4)
$$

$$
\begin{gathered}
\frac{\partial N_{i}^{0}}{\partial x}=\frac{b_{i}}{2 A}+\frac{b_{j}}{2 A}+\frac{\xi_{i} \eta_{i} g_{k}}{2 A\left(1+g_{1} g_{3}+g_{2} g_{4}\right)} S_{x}, \\
\frac{\partial N_{i}^{0}}{\partial y}=\frac{c_{i}}{2 A}+\frac{c_{j}}{2 A}+\frac{\xi_{i} \eta_{i} g_{k}}{2 A\left(1+g_{1} g_{3}+g_{2} g_{4}\right)} S_{y}, \\
\quad(i=1,2,3,4 ; j=2,3,4,1 ; k=3,4,1,2),
\end{gathered}
$$




$$
\begin{gathered}
S_{x}=\sum_{i^{\prime}=1}^{4} b_{i^{\prime}} \xi_{i^{\prime}} \eta_{i^{\prime}}\left[3\left(L_{j^{\prime}}-L_{m^{\prime}}\right)+\left(g_{j^{\prime}}-g_{k^{\prime}}\right)\right], \\
S_{y}=\sum_{i^{\prime}=1}^{4} c_{i^{\prime}} \xi_{i^{\prime}} \eta_{i^{\prime}}\left[3\left(L_{j^{\prime}}-L_{m^{\prime}}\right)+\left(g_{j^{\prime}}-g_{k^{\prime}}\right)\right], \\
\left(\begin{array}{l}
i^{\prime}=\overleftrightarrow{1,2,3,4} j^{\prime}=\overleftrightarrow{2,3,4,1} \\
\left.k^{\prime}=\overleftrightarrow{3,4,1,2} m^{\prime}=\overleftrightarrow{4,1,2,3}\right), \\
b_{i}=y_{j}-y_{k}, \quad c_{i}=x_{k}-x_{j}, \quad(\overleftrightarrow{i, j, k}=\overleftrightarrow{1,2,3,4})
\end{array}\right.
\end{gathered}
$$

in which $\left(x_{i}, y_{i}\right)(i=1,2,3,4)$ are the Cartesian coordinates of the four corner nodes.

2.2. The Additional Second-Order Displacement Interpolation Formulae. The additional displacements are assumed to be

$$
\begin{aligned}
& u_{\lambda}=\lambda_{1} L_{1} L_{3}+\lambda_{2} L_{2} L_{4}, \\
& v_{\lambda}=\lambda_{1}^{\prime} L_{1} L_{3}+\lambda_{2}^{\prime} L_{2} L_{4} .
\end{aligned}
$$

Thus, the c matrix $\mathbf{B}_{\lambda}$ is given by

$$
\begin{aligned}
& \mathbf{B}_{\lambda} \\
& =\left[\begin{array}{cccc}
\frac{b_{1} L_{3}+b_{3} L_{1}}{2 A} & 0 & \frac{b_{2} L_{4}+b_{4} L_{2}}{2 A} & 0 \\
0 & \frac{c_{1} L_{3}+c_{3} L_{1}}{2 A} & 0 & \frac{c_{2} L_{4}+c_{4} L_{2}}{2 A} \\
\frac{c_{1} L_{3}+c_{3} L_{1}}{2 A} & \frac{c b L_{3}+b_{3} L_{1}}{2 A} & \frac{c_{2} L_{4}+c_{4} L_{2}}{2 A} & \frac{b_{2} L_{4}+b_{4} L_{2}}{2 A}
\end{array}\right] .
\end{aligned}
$$

2.3. The Element Stiffness Matrix. Finally, the element stiffness matrix of the element can be expressed by

$$
\mathbf{K}^{e}=\mathbf{K}_{q q}-\mathbf{K}_{\lambda q}^{\mathrm{T}} \mathbf{K}_{\lambda \lambda}^{-1} \mathbf{K}_{\lambda q}
$$

with

$$
\begin{aligned}
& \mathbf{K}_{q q}=\iint_{A} \mathbf{B}_{q}^{\mathrm{T}} \mathbf{D} \mathbf{B}_{q} t \mathrm{~d} A, \\
& \mathbf{K}_{\lambda \lambda}=\iint_{A} \mathbf{B}_{\lambda}^{\mathrm{T}} \mathbf{D B}_{\lambda} t \mathrm{~d} A, \\
& \mathbf{K}_{\lambda q}=\iint_{A} \mathbf{B}_{\lambda}^{\mathrm{T}} \mathbf{D} \mathbf{B}_{q} t \mathrm{~d} A,
\end{aligned}
$$

where $t$ is the thickness of element; $\mathbf{D}$ is the elasticity matrix

$$
\mathbf{D}=\frac{E}{1-\mu^{2}}\left[\begin{array}{ccc}
1 & \mu & 0 \\
\mu & 1 & 0 \\
0 & 0 & \frac{1-\mu}{2}
\end{array}\right]
$$

where $E$ and $\mu$ are Young's modulus and Poisson's ratio, respectively. For plane strain problems, the $E$ and $\mu$ in (14) should be replaced by $E /\left(1-\mu^{2}\right)$ and $\mu /(1-\mu)$, respectively.
The resulting element model is the element AGQ6-I. It can be easily shown that the values of the additional displacement fields given by (10) are zero at four element nodes. However, without any other restrictions along element edges, element AGQ6-I is a nonconforming element. On the other hand, since the relationship between area and Cartesian coordinates is linear, the displacement fields of AGQ6-I are actually a second-order complete polynomial in Cartesian coordinates. This makes the element AGQ6-I insensitive to mesh distortion in higher-order problems. So, it cannot only present the exact solution for pure bending problem but also be free of MacNeal's trapezoid locking problem.

Unfortunately, as a nonconforming element like Wilson's Q6, element AGQ6-I cannot give the exact solutions for strict patch test either. Although the results can converge to the exact solutions by subdividing the mesh (weak patch test), further improvements on its convergence are valuable.

\section{Treatments on the Convergence of Element AGQ6-I}

3.1. Special Numerical Integration Scheme from QM6 [10]. In order to make the element Q6 [1] present exact solution for constant stress/strain problem with fixed coarse mesh, the following restriction condition was imposed by Taylor et al. [10] on its additional strain matrix:

$$
\int_{V^{e}} \mathbf{B}_{\lambda}^{\mathrm{T}} \mathrm{d} V \equiv \int_{-1}^{1} \int_{-1}^{1} \mathbf{B}_{\lambda}^{\mathrm{T}}|\mathbf{J}| t \mathrm{~d} \xi \mathrm{d} \eta=\mathbf{0} .
$$

For satisfying the previous equation, the value of determinant $|\mathbf{J}|$ of Jacobi matrix must be a constant. So, when evaluating the differentials of $x$ and $y$ to $\xi$ and $\eta$ in matrix $\mathbf{K}_{\lambda q}$ (see (13)) by numerical integration, the isoparametric coordinates of element centroid, $\xi=\eta=0$, were used to replace the coordinates of Gauss's integral points. Then, the value of $|\mathbf{J}|$ would be always a constant, and the resulting element, denoted by QM6, can pass the strict patch test.

In order to employ the previous technique to modify element AGQ6-I, the quadrilateral area coordinates can be rewritten in terms of the isoparametric coordinates:

$$
\begin{aligned}
L_{1} & =\frac{1}{4}(1-\xi)\left[g_{2}(1-\eta)+g_{3}(1+\eta)\right], \\
L_{2} & =\frac{1}{4}(1-\eta)\left[g_{4}(1-\xi)+g_{3}(1+\xi)\right], \\
L_{3} & =\frac{1}{4}(1+\xi)\left[g_{1}(1-\eta)+g_{4}(1+\eta)\right], \\
L_{4} & =\frac{1}{4}(1+\eta)\left[g_{1}(1-\xi)+g_{2}(1+\xi)\right] .
\end{aligned}
$$

Then, after substituting (16) into (11), the additional strain matrix $\mathbf{B}_{\lambda}$ of element AGQ6-I in (11) can be rewritten in terms of the isoparametric coordinates.

It is obvious that the additional strain matrix $\mathbf{B}_{\lambda}$ of element AGQ6-I is different with that of element Q6. For element Q6, there are only terms of $\xi, \eta$, and $\xi \eta$ available in $\mathbf{B}_{\lambda}$, and the integral interval of $(15)$ is $[-1,1]$. So long as 
the $|\mathbf{J}|$ is a constant, (15) will be satisfied, and the modified version of Q6, element QM6, can pass the strict patch test. Compared with Q6, the additional strain matrix $\mathbf{B}_{\lambda}$ of AGQ6I contains not only the terms of $\xi, \eta$, and $\xi \eta$ but also some constant terms which Q6 does not have. This leads to that the result of (15) is not a zero matrix. Therefore, the special numerical integration scheme mentioned previously cannot be directly used by element AGQ6-I. The influence of these constants must be considered. follows:

The constants in the additional strain matrix $\mathbf{B}_{\lambda}$ are as

$$
\begin{aligned}
& C_{1}=b_{1}\left(g_{1}+g_{4}\right)+b_{3}\left(g_{2}+g_{3}\right), \\
& C_{2}=b_{2}\left(g_{1}+g_{2}\right)+b_{4}\left(g_{3}+g_{4}\right), \\
& C_{3}=c_{1}\left(g_{1}+g_{4}\right)+c_{3}\left(g_{2}+g_{3}\right), \\
& C_{4}=c_{2}\left(g_{1}+g_{2}\right)+c_{4}\left(g_{3}+g_{4}\right) .
\end{aligned}
$$

In order to eliminate the influence of these constants, the modified additional strain matrix $\overline{\mathbf{B}}_{\lambda}$ of element AGQ6-I can be rewritten as

$$
\overline{\mathbf{B}}_{\lambda}=\mathbf{B}_{\lambda}-\left[\begin{array}{cccc}
C_{1} & 0 & C_{2} & 0 \\
0 & C_{3} & 0 & C_{4} \\
C_{3} & C_{1} & C_{4} & C_{2}
\end{array}\right]
$$

Using this modified additional strain matrix and the numerical integral method of QM6, the resulting element will pass the strict patch test.

The new model is named as AGQ6 M-I.

3.2. The Constant Stress Multiplier Method. The second approach is the constant stress multiplier method proposed by Pian and $\mathrm{Wu}[12]$, and can also make a nonconforming element pass the strict patch test. After introducing constant stress multiplier to eliminate the nonconforming energy on element sides, the final energy functional of the element can be written as

$$
\prod_{\mathrm{mp}}^{e}=\prod_{\mathrm{p}}^{e}-\oint_{\partial V^{e}} \boldsymbol{\sigma}_{C}^{\mathrm{T}} \mathbf{n}^{\mathrm{T}} \mathbf{u}_{\lambda} \mathrm{d} s
$$

Finally, the new form of the element stiffness matrix can be obtained as

$$
\mathbf{K}_{P}^{e}=\left[\begin{array}{cc}
\mathbf{K}_{q q} & \mathbf{K}_{q \lambda} \\
\mathbf{K}_{q \lambda}^{\mathrm{T}} & \mathbf{K}_{\lambda \lambda}
\end{array}\right]-\left[\begin{array}{cc}
\mathbf{0} & \mathbf{G} \\
\mathbf{G}^{\mathrm{T}} & \mathbf{0}
\end{array}\right],
$$

where

$$
\mathbf{G}=\int_{V^{e}} \mathbf{B}_{C}^{\mathrm{T}} \mathbf{D} \mathbf{B}_{\lambda} \mathrm{d} V
$$

in which $\mathbf{B}_{C}$ is the constant strain part of the matrix $\mathbf{B}_{q}$ given in (6).

The final element stiffness matrix can be derived by usual static condense procedure. The resulting new element is denoted by AGQ6 M-II and can also pass the strict form of patch test.
3.3. The Orthogonal Condition of Energy. In order to ensure the element to pass the strict patch test, the strain energy under constant stress state caused by the nonconforming strains is required to be zero:

$$
\int_{V^{e}} \boldsymbol{\sigma}_{c}^{\mathrm{T}} \boldsymbol{\varepsilon}_{\lambda} \mathrm{d} V=0
$$

where $\boldsymbol{\varepsilon}_{\lambda}=\mathbf{n}^{\mathrm{T}} \mathbf{u}_{\lambda}$ is the nonconforming strains of element. Equation (22) was called the orthogonal condition of energy in reference [29].

By eliminating the constant factor in (22), the patch test of constant stress/strain for any element can be rewritten as

$$
\oint_{\partial V^{e}} \mathbf{n}^{\mathrm{T}} \mathbf{u}_{\lambda} \mathrm{d} s=\int_{V^{e}} \varepsilon_{\lambda} \mathrm{d} V=0 .
$$

Equation (23) can be further decoupled into three cases corresponding to the states subjected to one stress component of $\sigma_{x}, \sigma_{y}$, and $\tau_{x y}$.

Thus, four constants $C_{i}(i=1,2,3,4)$ can be added to modify the additional strain field of AGQ6-I as follows:

$$
\begin{aligned}
\varepsilon_{x}= & \left(\frac{b_{1}}{2 A} L_{3}+\frac{b_{3}}{2 A} L_{1}+C_{1}\right) \lambda_{1} \\
& +\left(\frac{b_{2}}{2 A} L_{4}+\frac{b_{4}}{2 A} L_{2}+C_{2}\right) \lambda_{2}, \\
\varepsilon_{y}= & \left(\frac{c_{1}}{2 A} L_{3}+\frac{c_{3}}{2 A} L_{1}+C_{3}\right) \lambda_{1}^{\prime} \\
& +\left(\frac{c_{2}}{2 A} L_{4}+\frac{c_{4}}{2 A} L_{2}+C_{4}\right) \lambda_{2}^{\prime}, \\
\varepsilon_{x y}= & \left(\frac{c_{1}}{2 A} L_{3}+\frac{c_{3}}{2 A} L_{1}+C_{1}\right) \lambda_{1} \\
& +\left(\frac{c_{2}}{2 A} L_{4}+\frac{c_{4}}{2 A} L_{2}+C_{2}\right) \lambda_{2} \\
& +\left(\frac{b_{1}}{2 A} L_{3}+\frac{b_{3}}{2 A} L_{1}+C_{3}\right) \lambda_{1}^{\prime} \\
& +\left(\frac{b_{2}}{2 A} L_{4}+\frac{b_{4}}{2 A} L_{2}+C_{4}\right) \lambda_{2}^{\prime} .
\end{aligned}
$$

From $\int_{A^{e}} \varepsilon_{x} \mathrm{~d} A=0$, we obtain

$$
\begin{aligned}
\int_{A^{e}}[ & \left(\frac{b_{1}}{2 A} L_{3}+\frac{b_{3}}{2 A} L_{1}+C_{1}\right) \lambda_{1} \\
& \left.+\left(\frac{b_{2}}{2 A} L_{4}+\frac{b_{4}}{2 A} L_{2}+C_{2}\right) \lambda_{2}\right] \mathrm{d} A=0 .
\end{aligned}
$$

Its stronger forms can be written as

$$
\begin{aligned}
& \int_{\mathrm{A}^{e}}\left(\frac{b_{1}}{2 A} L_{3}+\frac{b_{3}}{2 A} L_{1}+C_{1}\right) \mathrm{d} A=0, \\
& \int_{\mathrm{A}^{e}}\left(\frac{b_{2}}{2 A} L_{4}+\frac{b_{4}}{2 A} L_{2}+C_{2}\right) \mathrm{d} A=0 .
\end{aligned}
$$




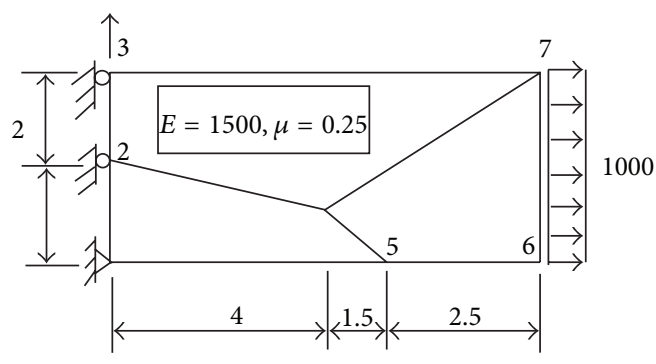

FIgURE 1: Patch test.

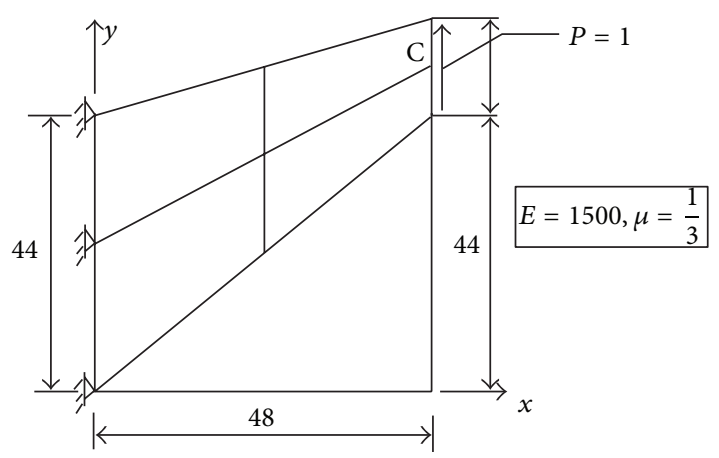

FIGURE 2: Cook's skew beam.

Then, $C_{1}$ and $C_{2}$ can be solved:

$$
\begin{aligned}
& C_{1}=-\frac{1}{6 A}\left[b_{1}\left(1-g_{2} g_{3}\right)+b_{3}\left(1-g_{4} g_{1}\right)\right], \\
& C_{2}=-\frac{1}{6 A}\left[b_{2}\left(1-g_{3} g_{4}\right)+b_{4}\left(1-g_{1} g_{2}\right)\right] .
\end{aligned}
$$

Similarly, from $\int_{\mathrm{A}^{e}} \varepsilon_{y} \mathrm{~d} A=0, C_{3}$ and $C_{4}$ can be obtained:

$$
\begin{aligned}
& C_{3}=-\frac{1}{6 A}\left[c_{1}\left(1-g_{2} g_{3}\right)+c_{3}\left(1-g_{4} g_{1}\right)\right], \\
& C_{4}=-\frac{1}{6 A}\left[c_{2}\left(1-g_{3} g_{4}\right)+c_{4}\left(1-g_{1} g_{2}\right)\right] .
\end{aligned}
$$

And it can be proved that all previous $C_{i}(i=1,2,3,4)$ satisfy $\int_{\mathrm{A}^{e}} \varepsilon_{x y} \mathrm{~d} A=0$ automatically.

Substitution of (27) and (28) into (24) yields the additional strain matrix of a new element by modifying element AGQ6-I. This new model is denoted by AGQ6 M.

\section{Numerical Examples}

4.1. Strict Patch Test. The constant strain/stress patch test using irregular mesh is shown in Figure 1. Let Young's modulus $E=1000$, Poisson's ratio $\mu=0.25$, and thickness of the patch $t=1$. Element AGQ6-I cannot give the exact solutions under such coarse meshes, while all three new elements with full integration scheme $(2 \times 2)$ can produce exact solutions without any problem. It shows that the proposed three methods are all workable for modifying nonconforming elements.

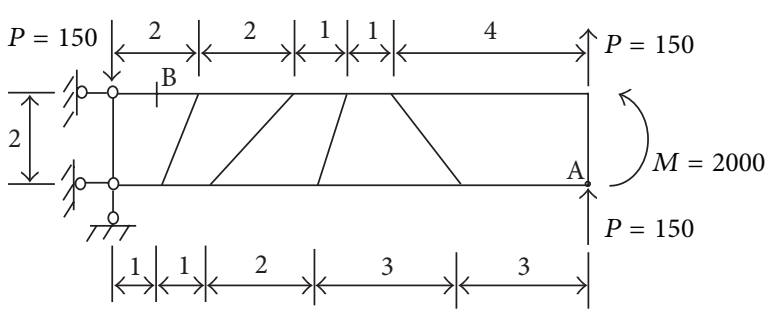

Figure 3: Cantilever beam with five irregular elements.

TABle 1: Displacement at point C of Cook's beam.

\begin{tabular}{lccc}
\hline Element & & $V_{\mathrm{C}}$ & \\
& $2 \times 2$ & $4 \times 4$ & $8 \times 8$ \\
\hline Q4 & 11.80 & 18.29 & 22.08 \\
Q6 & 22.94 & 23.48 & 23.80 \\
QM6 & 21.05 & 23.02 & - \\
AGQ6-I & 23.06 & 23.68 & 23.87 \\
AGQ6M-I & 20.86 & 23.00 & 23.69 \\
AGQ6M-II & 11.75 & 18.28 & 22.08 \\
AGQ6M & 20.74 & 22.99 & 23.69 \\
\hline Reference value & & 23.96 & \\
\hline
\end{tabular}

TABLE 2: The deflections at point $\mathrm{A}$ of a cantilever beam.

\begin{tabular}{lcccc}
\hline \multirow{2}{*}{ Element type } & \multicolumn{2}{c}{ Load (a) } & \multicolumn{2}{c}{ Load (b) } \\
& $v_{\mathrm{A}}$ & $\sigma_{x \mathrm{~B}}$ & $v_{\mathrm{A}}$ & $\sigma_{x \mathrm{~B}}$ \\
\hline Q4 & 45.7 & -1761 & 50.7 & -2448 \\
Q6 & 98.4 & -2428 & 100.4 & -3354 \\
QM6 & 96.1 & -2497 & 98.0 & -3235 \\
QC6 & 96.1 & -2439 & 98.1 & -3339 \\
NQ6 & 96.1 & -2439 & 98.0 & -3294 \\
AGQ6-I & 100.0 & -3000 & 102.0 & -4151 \\
AGQ6M-I & 91.9 & - & 94.5 & - \\
AGQ6M-II & 44.4 & - & 49.4 & - \\
AGQ6M & 96.0 & -3015 & 97.9 & -4135 \\
\hline Exact & 100 & -3000 & 102.6 & -4050 \\
\hline
\end{tabular}

4.2. Cook's Skew Beam. This example was proposed by Cook et al. [9]. As shown in Figure 2, a skew cantilever beam is subjected to distributed shear load along its free edge. The results of vertical deflection at point $\mathrm{C}$ are listed in Table 1.

Compared with the other elements, the accuracy of AQ6 M-II is even poor as Q4 element, while AGQ6 M-I and AGQ6 $\mathrm{M}$ are as accurate as QM6.

4.3. Cantilever Beam Divided by Five Quadrilateral Elements. The cantilever beam, as shown in Figure 3, is divided by five irregular quadrilateral elements. Two loading cases are considered: (a) pure bending under moment $M$; (b) linear bending under transverse force $P$. The Young's modulus $E=$ 1500 , and Poisson's ratio $\mu=0.25$. Numerical results of the vertical deflection $v_{\mathrm{A}}$ at point $\mathrm{A}$ and the stress $\sigma_{x \mathrm{~B}}$ at point $\mathrm{B}$ are given in Table 2.

From the results listed in Table 2, AGQ6 M-II still exhibits poor performance as Q4. Although AGQ6 M-I has passed 


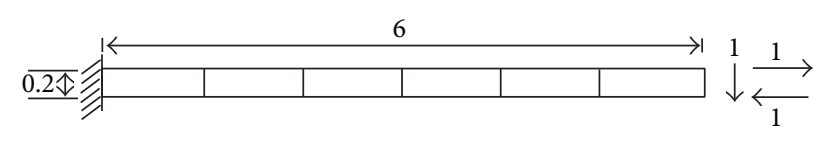

(a)

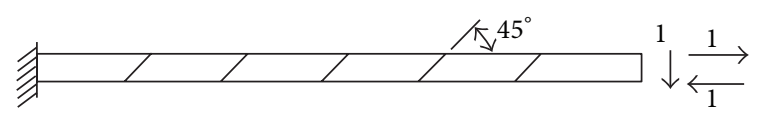

(b)

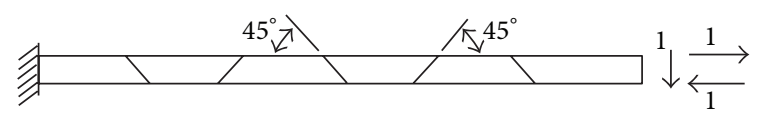

(c)

FIGURE 4: MacNeal's thin beam.

TABLE 3: The normalized deflections at the free end of MacNeal's beam.

\begin{tabular}{lcccccc}
\hline $\begin{array}{l}\text { Element } \\
\text { type }\end{array}$ & $\begin{array}{c}\text { Mesh } \\
(\mathrm{a})\end{array}$ & $\begin{array}{c}\text { Mesh } \\
(\mathrm{b})\end{array}$ & $\begin{array}{c}\text { Mesh } \\
(\mathrm{c})\end{array}$ & $\begin{array}{c}\text { Mesh } \\
(\mathrm{a})\end{array}$ & $\begin{array}{c}\text { Mesh } \\
(\mathrm{b})\end{array}$ & $\begin{array}{c}\text { Mesh } \\
(\mathrm{c})\end{array}$ \\
\hline Q4 & 0.093 & 0.035 & 0.003 & 0.093 & 0.031 & 0.022 \\
Q6 & 0.993 & 0.677 & 0.106 & 1.000 & 0.759 & 0.093 \\
QM6 & 0.993 & 0.623 & 0.044 & 1.000 & 0.722 & 0.037 \\
AGQ6-I & 0.993 & 0.994 & 0.994 & 1.000 & 1.000 & 1.000 \\
AGQ6M-I & 0.993 & 0.631 & 0.050 & 1.000 & 0.722 & 0.044 \\
AGQ6M-II & 0.093 & 0.034 & 0.027 & 0.093 & 0.031 & 0.022 \\
AGQ6M & 0.993 & 0.632 & 0.051 & 1.000 & 0.726 & 0.046 \\
\hline Exact & & -0.1081 & & & -0.0054 & \\
\hline
\end{tabular}

the patch test, it suffers from the mesh distortion now; only AGQ6 M can keep the same accuracy as other elements.

4.4. MacNeal's Thin Cantilever Beam with Distorted Meshes. Consider the thin beams presented in Figure 4. Three different mesh shapes, rectangular, parallelogram and trapezoidal, are adopted. This example, proposed by MacNeal and Harder [20], is a famous benchmark for testing the sensitivity to mesh distortion of quadrilateral membrane elements. In the three kinds of mesh, the trapezoidal shapes will cause the element locking easily.

There are two loading cases under consideration: pure bending and transverse linear bending. The Young's modulus of the beam $E=10^{7}$; the Poisson's ratio $\mu=0.3$; and the thickness of the beam $t=0.1$.

The results of the tip deflection are shown in Table 3. From the results listed in Table 3,

(1) both AGQ6 M-II and Q4 suffer from locking problems for the three kinds of mesh distortions ((a) length-width ratio distortion, (b) parallelogram distortion, and (c) trapezoidal distortion) of three different meshes.

(2) As a nonconforming element, Q6 still cannot overcome the locking problem for the trapezoidal mesh. Benefited from the area coordinate methods, AGQ6-I is locking free from each kind of mesh.

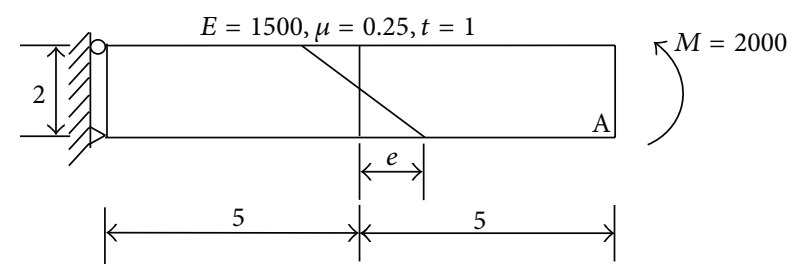

FIGURE 5: Cantilever beam divided by two elements with distorted parameter $e$.

TABLE 4: Results of the tip deflection of a cantilever beam subjected to a pure bending $M$.

\begin{tabular}{lccccccc}
\hline$e$ & 0 & 0.5 & 1 & 2 & 3 & 4 & 4.9 \\
\hline Q4 & 28.0 & 21.0 & 14.1 & 9.7 & 8.3 & 7.2 & 6.2 \\
Q6 & 100 & 78.0 & 56.1 & 42.5 & 41.5 & 44.2 & 47.4 \\
QM6 & 100 & 80.9 & 62.7 & 54.4 & 53.6 & 51.2 & 46.8 \\
AGQ6-I & 100 & 100 & 100 & 100 & 100 & 100 & 100 \\
AGQ6M-I & 100 & 80.8 & 55.4 & 24.1 & 12.9 & 9.4 & 7.6 \\
AGQ6M-II & 28.0 & 21.2 & 14.1 & 9.2 & 7.3 & 5.9 & 4.9 \\
AGQ6M & 100 & 83.8 & 66.5 & 60.1 & 61.4 & 60.3 & 56.0 \\
\hline Exact & 100 & 100 & 100 & 100 & 100 & 100 & 100 \\
\hline
\end{tabular}

(3) All those elements which can pass the strict patch test are locked in the trapezoidal mesh distortion. This verified the theory proposed in [30] again; that is, the trapezoidal locking is unavoidable for an element passed the strict patch test.

4.5. Mesh Distortion. The cantilever beam shown in Figure 5 is divided by two elements. The shape of the two elements varies with the variety of the distorted parameter $e$. When $e=$ 0 , both elements are rectangular. But with the increase of $e$, the mesh will be distorted more and more seriously. This is another famous benchmark for testing the sensitivity to the mesh distortion. Pure bending $M=2000$ is considered. The results of the tip deflection at point $\mathrm{A}$ are listed in Table 4.

The accuracy of element Q4 and AGQ6 M-II is the poorest. Their relative error reaches $72 \%$ when $e=0$. Except Q4, AGQ6 M-I is much more sensitive to mesh distortion than any other element although it can present exact solution while $e=0$. Compared with other elements which can pass the strict patch test, AGQ6 $\mathrm{M}$ is the most robust element against mesh distortion.

\section{Conclusions}

In order to make the nonconforming element AGQ6-I pass the strict patch test, three treatments are used and tested. These methods are special numerical integration scheme used in QM6, constant stress multiplier method, and the orthogonal condition of energy. Numerical results of numerical examples show that each method can achieve the goal, but the performance for complicated stress problem of new elements will degenerate inevitably. Among the three treatments, the constant stress multiplier method made the element so rigid that the accuracy will deteriorate even in rectangular meshes. The special numerical integration scheme can make element 
QM6 exhibit good performance, but it is not always suitable for all other elements. The orthogonal condition of energy can effectively improve the compatibility of element AGQ6-I without losing too much accuracy.

\section{Acknowledgments}

The authors would like to acknowledge the financial supports of the National Natural Science Foundation of China (11272181), the Specialized Research Fund for the Doctoral Program of Higher Education of China (20120002110080), and the National Basic Research Program of China (Project no. 2010CB832701).

\section{References}

[1] E. L. Wilson, R. L. Taylor, W. P. Doherty, and T. Ghabussi, "Incompatible displacement models," in Numerical Computational Methods in Structural Mechanics, S. T. Fenven et al., Ed., pp. 43-57, Academic Press, New York, NY, USA, 1973.

[2] R. J. Melosh, "Basis for derivation of matrices for the direct stiffness method," AIAA Journal, vol. 1, no. 7, pp. 1631-1637, 1963.

[3] G. P. Bazeley, Y. K. Cheung, B. M. Irons, and O. C. Zienkiewicz, "Triangular element in plate bending-conforming and nonconforming solutions," in Proceedings of the 1st Conference on Matrix Method in Structural Mechanics, pp. 547-576, Wright Patterson Air Force Base, Dayton, Ohio, USA, 1965.

[4] B. M. Irons and A. Razzaque, "Experience with the patch test for convergence of finite elements methods," in Mathematical Foundations of the Finite Element Methods, pp. 557-587, Academic Press, New York, NY, USA, 1972.

[5] O. C. Zienkiewicz and R. L. Taylor, The Finite Element Method. Vol. 1. The basis, Butterworth-Heinemann, Oxford, UK, 5th edition, 2000.

[6] G. Strang and G. J. Fix, An Analysis of the Finite Element Method, Prentice Hall, Englewood Cliffs, NJ, USA, 1973.

[7] F. Stummel, "The limitation of the patch test," International Journal for Numerical Methods in Engineering, vol. 15, no. 2, pp. 177-188, 1980.

[8] Z. C. Shi, "An explicit analysis of Stummel's patch test examples," International Journal for Numerical Methods in Engineering, vol. 20, no. 7, pp. 1233-1246, 1984.

[9] R. D. Cook, D. S. Malkus, and M. E. Plesha, Concepts and Applications of Finite Element Analysis, John Wiley \& Sons, New York, NY, USA, 3rd edition, 1989.

[10] R. L. Taylor, P. J. Beresford, and E. L. Wilson, "A non-conforming element for stress analysis," International Journal For Numerical Methods in Engineering, vol. 10, pp. 1211-1219, 1976.

[11] E. L. Wachspress, "Incompatible quadrilateral basis functions," International Journal For Numerical Methods in Engineering, vol. 12, pp. 589-595, 1978.

[12] T. H. H. Pian and C.-C. Wu, Hybrid and Incompatible Finite Element Methods, Chapman \& Hall, Boca Raton, Fla, USA, 2006.

[13] Y.-Q. Long and M.-F. Huang, "A generalized conforming isoparametric element," Applied Mathematics and Mechanics, vol. 9, no. 10, pp. 929-936, 1988.

[14] W. J. Chen and L. M. Tang, "Isoparametric quasi-conforming element," Journal of Dalian University of Technology, vol. 20, no. 1, pp. 63-74, 1981.
[15] T. H. H. Pian and K. Sumihara, "Rational approach for assumed stress finite elements," International Journal for Numerical Methods in Engineering, vol. 20, no. 9, pp. 1685-1695, 1984.

[16] S. Cen, T. Zhang, C.-F. Li, X.-R. Fu, and Y.-Q. Long, "A hybridstress element based on Hamilton principle," Acta Mechanica Sinica, vol. 26, no. 4, pp. 625-634, 2010.

[17] X.-M. Chen, S. Cen, Y.-Q. Long, and Z.-H. Yao, "Membrane elements insensitive to distortion using the quadrilateral area coordinate method," Computers and Structures, vol. 82, no. 1, pp. 35-54, 2004.

[18] Y. Q. Long, J. X. Li, Z. F. Long, and S. Cen, "Area co-ordinates used in quadrilateral elements," Communications in Numerical Methods in Engineering, vol. 15, no. 8, pp. 533-545, 1999.

[19] Z. F. Long, J. X. Li, S. Cen, and Y. Q. Long, "Some basic formulae for area co-ordinates in quadrilateral elements," Communications in Numerical Methods in Engineering, vol. 15, no. 12, pp. 841-852, 1999.

[20] R. H. MacNeal and R. L. Harder, "A proposed standard set of problems to test finite element accuracy," Finite Elements in Analysis and Design, vol. 1, no. 1, pp. 3-20, 1985.

[21] Y. Du and S. Cen, "Geometrically nonlinear analysis with a 4node membrane element formulated by the quadrilateral area coordinate method," Finite Elements in Analysis and Design, vol. 44, no. 8, pp. 427-438, 2008.

[22] S. Cen, Y. Du, X.-M. Chen, and X.-R. Fu, “The analytical element stiffness matrix of a recent 4-node membrane element formulated by the quadrilateral area co-ordinate method," Communications in Numerical Methods in Engineering, vol. 23, no. 12, pp. 1095-1110, 2007.

[23] R. P. R. Cardoso, J. W. Yoon, and R. A. F. Valente, "A new approach to reduce membrane and transverse shear locking for one-point quadrature shell elements: linear formulation," International Journal for Numerical Methods in Engineering, vol. 66, no. 2, pp. 214-249, 2006.

[24] R. P. R. Cardoso, J. W. Yoon, and R. A. F. Valente, "Enhanced one-point quadrature shell element for nonlinear applications," International Journal for Numerical Methods in Engineering, vol. 69, no. 3, pp. 627-663, 2007.

[25] J. W. Yoon, R. P. R. Cardoso, and R. E. Dick, "Puncture fracture in an aluminum beverage can," International Journal of Impact Engineering, vol. 37, no. 2, pp. 150-160, 2010.

[26] G. Prathap and V. Senthilkumar, "Making sense of the quadrilateral area coordinate membrane elements," Computer Methods in Applied Mechanics and Engineering, vol. 197, no. 49-50, pp. 4379-4382, 2008.

[27] R. Flajs, S. Cen, and M. Saje, "On convergence of nonconforming convex quadrilateral finite elements AGQ6," Computer Methods in Applied Mechanics and Engineering, vol. 199, no. 2528, pp. 1816-1827, 2010.

[28] R. P. R. Cardoso and J.-W. Yoon, "One point quadrature shell elements: a study on convergence and patch tests," Computational Mechanics, vol. 40, no. 5, pp. 871-883, 2007.

[29] P. G. Bergan and M. K. Nygard, "Finite element with increased freedom in choosing shape function," International Journal for Numerical Methods in Engineering, vol. 20, no. 4, pp. 643-663, 1984.

[30] R. H. MacNeal, "A theorem regarding the locking of tapered four-noded membrane elements," International Journal for Numerical Methods in Engineering, vol. 24, no. 9, pp. 1793-1799, 1987. 


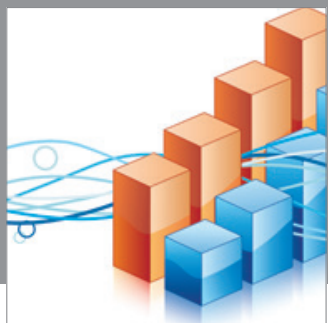

Advances in

Operations Research

mansans

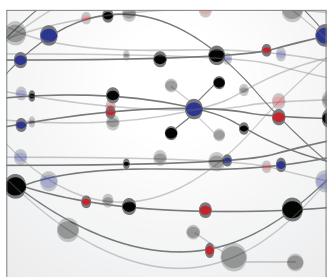

The Scientific World Journal
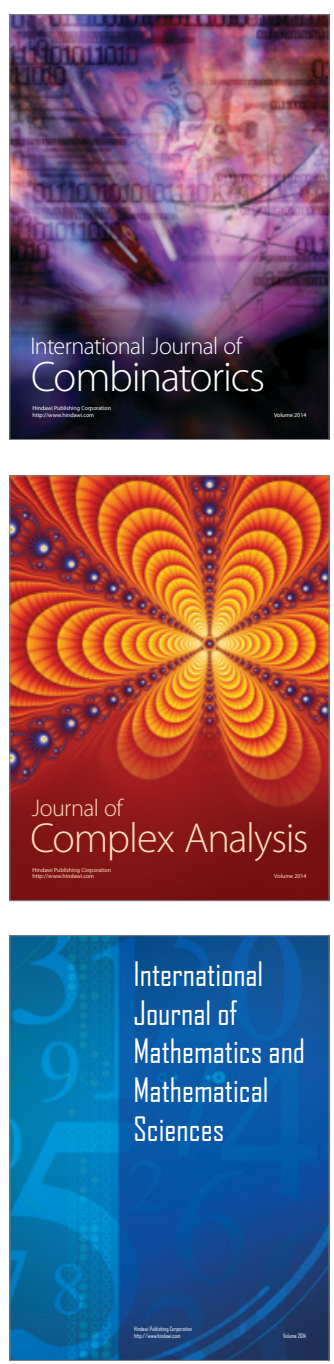
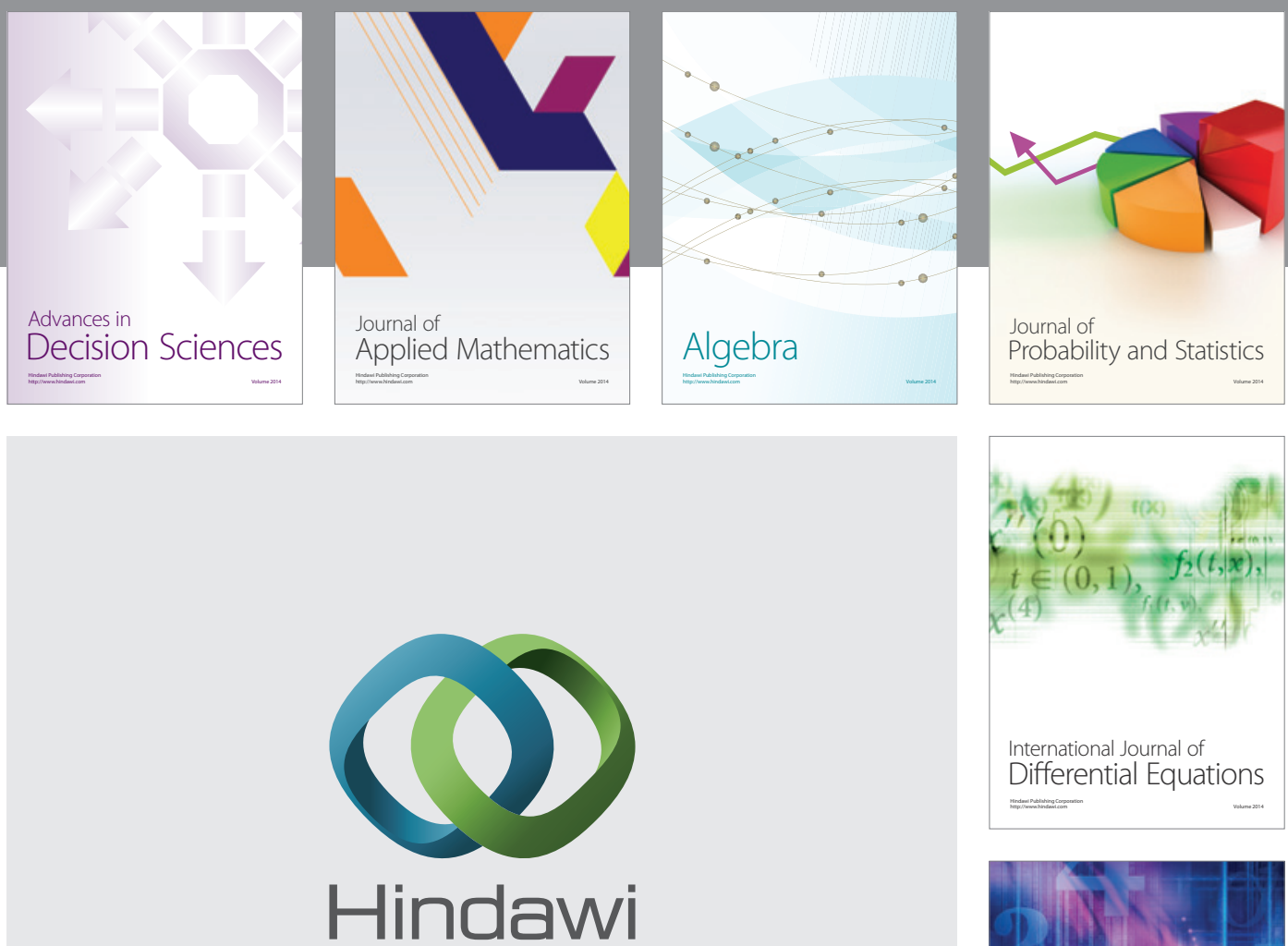

Submit your manuscripts at http://www.hindawi.com
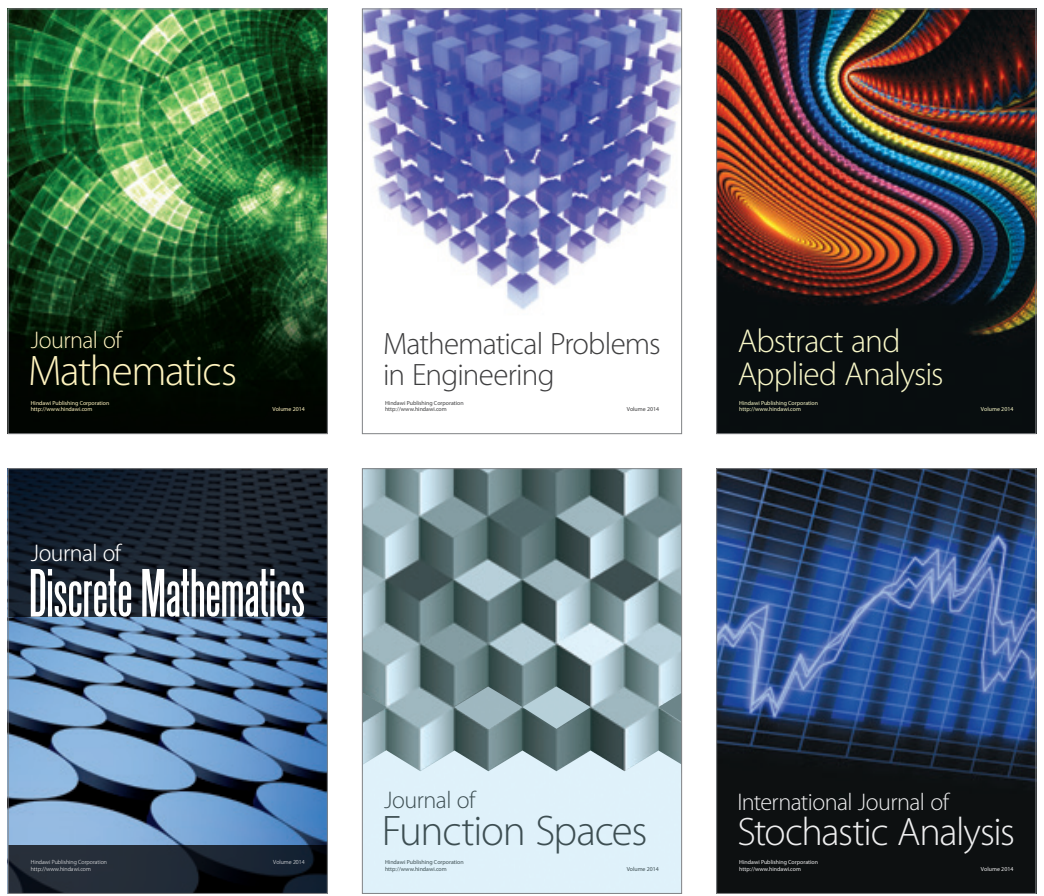

Journal of

Function Spaces

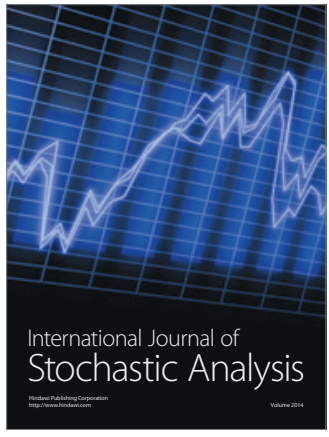

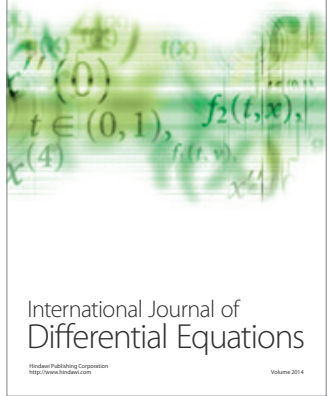
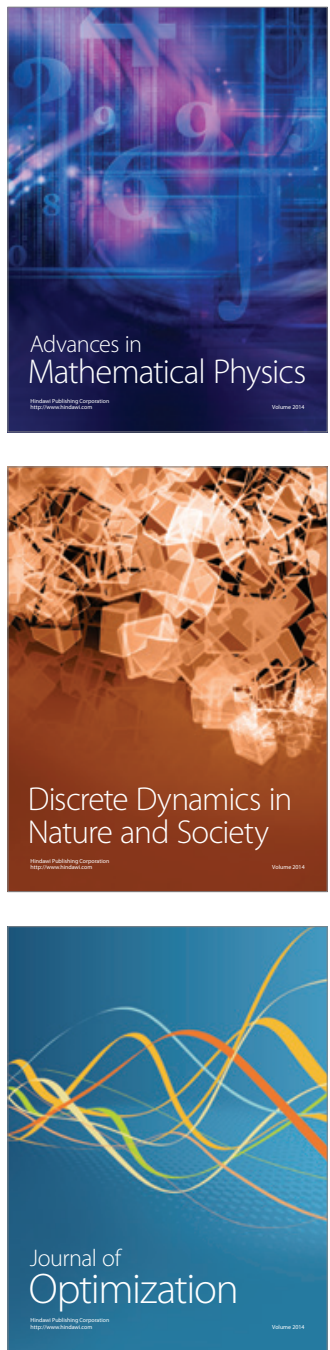